\title{
Analysis of segregation and bifurcation in turbulent spray flames: a 3D counterflow configuration
}

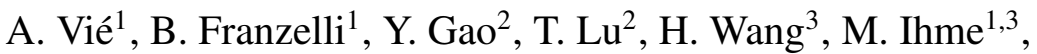 \\ ${ }^{1}$ Center for Turbulence Research, Stanford University, Stanford, California 94305, USA \\ ${ }^{2}$ Department of Aerospace and Mechanical Engineering, University of Southern California, Los Angeles, California 90089, USA \\ ${ }^{3}$ Department of Mechanical Engineering, Stanford University, Stanford, CA 94305, USA
}

\section{CORRESPONDING AUTHOR:}

Name: A. Vié

Address: Center for Turbulence Research, 488 Escondido Mall,

Stanford University,

Stanford, CA 94305-3024, USA

Phone: (+1)650-736-0766

Fax: $(+33) 650-725-3525$

e-mail: aymeric.vie@stanford.edu

\section{COLLOQUIUM:}

Colloquium: Spray and droplet combustion

\section{PAPER LENGTH:}

Abstract $($ Method 1$)=\mathbf{1 8 3}$ words

Total Main Paper (Method 2) : 6125 words

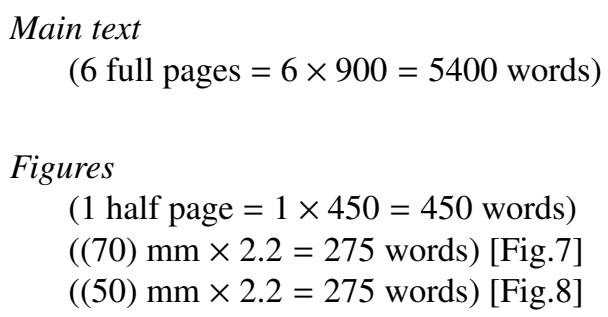

( 1 half page $=1 \times 450=450$ words $)$

((70) $\mathrm{mm} \times 2.2=275$ words) [Fig.7]

$((50) \mathrm{mm} \times 2.2=275$ words) [Fig.8] 


\title{
Analysis of segregation and bifurcation in turbulent spray flames: a 3D counterflow configuration
}

\author{
Aymeric Viéa ${ }^{\mathrm{a}}$ Benedetta Franzellia ${ }^{\mathrm{a}}$ Yang Gao ${ }^{\mathrm{b}}$, Tianfeng Lu ${ }^{\mathrm{b}}$, Hai Wang ${ }^{\mathrm{c}}$, Matthias Ihme $^{\mathrm{a}, \mathrm{c}}$ \\ ${ }^{a}$ Center for Turbulence Research, Stanford University, Stanford, CA 94305, USA \\ ${ }^{b}$ Department of Mechanical Engineering, University of Connecticut, Storrs, CT 06269, USA \\ ${ }^{c}$ Department of Mechanical Engineering, Stanford University, Stanford, CA 94305, USA
}

\begin{abstract}
The understanding of spray combustion processes is of primary importance, as it is encountered in a wide range of industrial applications. In the present work, mesoscale-resolved simulations of a 3D turbulent counterflow spray configuration are conducted. Primary focus is on examining the effect of the coupling between turbulence, evaporation, mixing, and combustion. By considering different initial droplet diameters and through comparisons with turbulent and laminar configurations at the same operating condition, it is shown that preferential concentration can lead to conditions of locally high mixture-fraction composition. In addition, local variability in strain rate and droplet diameter introduces a bifurcation of the spray flame. This bifurcation consists of spray flame structures exhibiting single-reaction or double-reaction structures. It is shown that this bimodal behavior is linked to the existence of a hysteresis in the laminar spray flame structure for droplet diameter variations, as well as the occurrence of a bifurcation for strain rate variations. These results have direct implications for flamelet-based tabulation methods, since identifying the appropriate flamelet structure in turbulent spray flames would require informations about boundary conditions and the flamelet history.
\end{abstract}

Keywords: spray flame; 3D counterflow; turbulence; bifurcation; hysteresis

Email addresses: aymeric.vie@stanford.edu (Aymeric Vié), franzelb@stanford.edu (Benedetta Franzelli), yang.gao@uconn.edu (Yang Gao), tlu@engr .uconn. edu (Tianfeng Lu), hai . wang@stanford.edu (Hai Wang), mihme@stanford.edu (Matthias Ihme) 


\section{Introduction}

Because of high energy density of liquid fuel, spray combustion is one of the most common mechanisms to convert liquid fuels into thermal energy in industrial applications. In aeronautical combustors, the spray is usually injected into the combustion chamber at the exit of a swirl stage. The main function of this swirl stage is to enhance the mixing as well as to shorten the flame by limiting its axial expansion. In such systems, the interaction between the spray, the flame and the flow field is highly intricate, and the overall behavior and stabilization processes that take place are the consequence of these complex mechanisms. Consequently, fundamental investigations are needed to understand the underlying physical processes that are associated with the coupling between turbulence, evaporation, mixing, and combustion $[1,2,3]$.

At laminar conditions, spray, mixing and combustion are coupled by mutual interactions: the spray evaporation generates the gaseous fuel which mixes with air into a flammable mixture. This gaseous reactive mixture feeds the flame which in turn heats the droplets and thus sustains fuel supply. Several studies have been devoted to the investigation of these interactions, see $[4,5]$ and references therein for exhaustive parametric studies. In turbulent flows, the physics becomes even more intricate: the turbulence may act on the spray, by inducing preferential concentration, which modifies the local mixture fraction field. In addition, the interaction of the turbulence with the flame results in wrinkling and stretching of the flame.

Several studies have investigated 2D unsteady configurations $[6,7,8]$, highlighting preferential concentration effects and the influence of local strain rate. A 3D swirled spray flame has also been investigated in [9], showing the coexistence of premixed and diffusion regime, and the role of rich premixed zones on flame stabilization. The goal of the present work is to investigate the structure of turbulent spray flames, by considering a 3D statistically stationary counterflow system. Compared to previous studies, in which global one-step reaction mechanisms have been used to describe the chemistry, a 24-species reduced mechanism for n-dodecane is used, which provides an accurate representation of the chemical phenomena.

The remainder of this paper is organized as follows. In Sec. 2, the modeling approach and the numerics are briefly summarized. The 3D turbulent counterflow configuration, and all relevant non-dimensional parameters that characterize the configuration are summarized in Sec. 3. Results for monodispersed spray injection and different droplet diameters are presented in Sec. 4. The focus of the analysis is on characterizing the bimodal flame structure and on describing the hysteresis and the spray-flame bifurcations. The paper finishes with conclusions. 


\section{Modeling and numerics}

\subsection{Gas-phase equations}

The gas phase is described by the conservation equations for mass, momentum, species, and energy:

$$
\begin{aligned}
\mathrm{D}_{t} \rho & =\dot{S}_{m}, \\
\mathrm{D}_{t}\left(\rho u_{i}\right) & =\dot{S}_{u_{i}}-\partial_{i} p+\partial_{j}\left(\sigma_{i j}\right), \\
\mathrm{D}_{t}\left(\rho Y_{k}\right) & =\partial_{j}\left(\rho D_{k} \frac{W_{k}}{W} \partial_{j} X_{k}\right)+\dot{\omega}_{k}+\dot{S}_{m} \delta_{k F}, \\
\mathrm{D}_{t}(\rho T) & =\partial_{j}\left(\frac{\lambda}{c_{p}} \partial_{j} T\right)+\frac{\lambda}{c_{p}^{2}} \partial_{j} T \partial_{j} c_{p}-\sum_{k=1}^{N_{s}} \frac{h_{k} \dot{\omega}_{k}}{c_{p}}+\dot{S}_{T},
\end{aligned}
$$

where $\mathrm{D}_{t} \phi=\partial_{t} \phi+\partial_{i} u_{i} \phi, \partial_{i} \equiv \partial_{x_{i}}, \rho$ is the gas density, $u_{i}$ the gas velocity, $\dot{S}_{m}, \dot{S}_{u_{i}}$, and $\dot{S}_{T}$ the source terms due to

droplet evaporation, drag force, and heat transfer. The pressure is denoted by $p$, and $\sigma_{i j}=\mu\left[\partial_{i} u_{j}+\partial_{j} u_{i}-\frac{2}{3} \partial_{k} u_{k} \delta_{i j}\right]$ is the viscous stress tensor. The mass fraction and mole fraction of species $k$ are denoted by $Y_{k}$ and $X_{k}$, respectively. The molecular weight of species $k$ is denoted by $W_{k}$ and $W$ is the mixture-averaged molecular weight. The diffusivity of species $k$ is denoted by $D_{k}, \dot{\omega}_{k}$ is the reaction source term of species $k$, and $\delta_{k F}$ is the Kronecker function that is unity for fuel and zero for all other species. The temperature is denoted by $T, \lambda$ is the thermal conductivity, $c_{p}$ is the heat capacity, and $h_{k}$ is the enthalpy of species $k$.

\subsection{Dispersed-phase equations}

For the dispersed phase, a Lagrangian point-particle approach is used (see [10] for details). The equations describing each droplet are written as:

$$
\begin{aligned}
\mathrm{d}_{t} x_{d, i} & =u_{d, i}, \\
\mathrm{~d}_{t} u_{d, i} & =f_{d, i}=\frac{f_{1}}{\tau_{d}}\left[u_{i}\left(\mathbf{x}_{\mathbf{d}}\right)-u_{d, i}\right], \\
\mathrm{d}_{t} T_{d} & =\dot{T}_{d}=\frac{\mathrm{Nu}}{3 \operatorname{Pr}} \frac{c_{p}}{c_{l}} \frac{f_{2}}{\tau_{d}}\left[T\left(\mathbf{x}_{\mathbf{d}}\right)-T_{d}\right]+\frac{\dot{m}_{d} l_{v}}{m_{d} c_{l}}, \\
\mathrm{~d}_{t} m_{d} & =\dot{m}_{d}=-\frac{\operatorname{Sh}}{3 \operatorname{Sc}} \frac{m_{d}}{\tau_{d}} \ln \left(1+B_{M}\right),
\end{aligned}
$$

where $\boldsymbol{x}_{d}$ is the position of the droplet, $\boldsymbol{u}_{d}$ its velocity, $T_{d}$ its temperature and $m_{d}$ its mass. Nu is the Nusselt number, Pr the Prandtl number, Sh the Sherwood number, Sc the Schmidt number, and $B_{M}$ the Spalding number. The relaxation time of the droplet is $\tau_{d}=\rho_{l} d^{2} / 18 \mu, \rho_{l}$ its density, $d$ its diameter, $c_{l}$ its heat capacity and $l_{v}$ the latent heat of 
vaporization. The drag factor is $f_{1}$, accounting for high Reynolds number effects and $f_{2}$ is a correction factor to account for effects of heat-exchange on the evaporation [10].

The coupling terms with the gas phase are obtained by integrating the contributions from all droplets contained in the control volume $\Delta V$ :

$$
\begin{aligned}
& \dot{S}_{m}=-\left\{\mathrm{d}_{t} m_{d}\right\}, \\
& \dot{S}_{u_{i}}=-\left\{\mathrm{d}_{t} m_{d} u_{d, i}\right\}, \\
& \dot{S}_{T}=-\frac{1}{c_{p}}\left\{c_{l} m_{d} \mathrm{~d}_{t} T_{d}+\left(c_{p} T_{d}+l_{v}\right) \mathrm{d}_{t} m_{d}\right\},
\end{aligned}
$$

where $\{\cdot\}=\frac{1}{\Delta V} \sum_{d \in \Delta V}$.

\subsection{Reaction chemistry}

In the present study, a 24-species mechanism for n-dodecane is used, which is based on the JetSurF 1.0-11 mechanism [11], originally consisting of 123 species and 977 reactions. JetSurF 1.0-11 is a simplified version of JetSurF 1.0. It features a lumped model for n-alkane cracking and the detailed USC Mech II [12] for the pyrolysis and oxidation of C1-C4 hydrocarbons. The pyrolysis of n-dodecane is described by a lumped model of three species for cracking to form $\mathrm{C} 1-\mathrm{C} 4$ fragments [13]. The lumping presents a considerable simplification over the detailed chemistry in JetSurF, but it retains the accuracy of the full model [11] over a wide range of conditions. In the present work, the USC Mech II part of the mechanism was systematically reduced with directed relation graph (DRG), DRG-aided sensitivity analysis, and linearized quasi steady state (QSS) approximations [14]. The reduced mechanism is validated in auto-ignition and perfectly stirred reactors (PSR), and results for stochiometric conditions are shown in Fig. 1. Similar agreement was also achieved for equivalence ratios of 0.5 and 1.5. Detailed thermodynamic and transport properties are considered. The species diffusivities $D_{k}$ are calculated assuming constant but not equal species Lewis number Le ${ }_{k}$, i.e. $D_{k}=\lambda\left(\rho c_{p} \mathrm{Le}_{k}\right)$, provided as a supplementary material together with the 24-species mechanism.

\subsection{Numerics}

The governing equations are solved in the low Mach number limit using the structured 3DA code [15]. A QUICK scheme is used for the discretization of the scalar advection operators, and a second-order central scheme is used for solving the momentum and pressure equations, in combination with the HYPRE library for solving the Poisson equation. A staggered representation is used where the velocity is defined at the cell face, while the scalars are located at the cell center. Time-integration is performed using a second-order Crank-Nicholson scheme. The chemical source terms are evaluated using the DVODE library, which uses an adaptive time stepping to advance the system of ODEs. 


\section{Three-dimensional turbulent spray counterflow configuration}

\subsection{Setup of the configuration}

We consider a 3D counterflow configuration which consists of two opposed square slots. The separation distance of $L=0.02 m$ is selected to be comparable to experiments, see [16]. The direction $x_{1}=x$ is the injection direction, the direction $y=x_{2}$ the outflow, and the direction $z=x_{3}$ is periodic. The mesh consists in $256 \times 384 \times 256$ cells. Grid-convergence studies were performed to confirm that this resolution is adequate for resolving the flame structure and all turbulence scales.

At the fuel side, pure air is injected with a fuel spray composed of n-dodecane at ambient conditions $\left(T_{d}^{F}=T^{F}=\right.$ $300 \mathrm{~K})$. Here and in the following the superscripts " $\mathrm{F}$ " and "O" refer to the fuel side and the oxidizer side, respectively. The equivalence ratio is $\phi=s \frac{\dot{m}_{F}}{\dot{m}_{O}}=2.0$ (where $s$ is the stoichiometric mass ratio), and the corresponding liquid mass flow rate is $6.18 \times 10^{-4} \mathrm{~kg} / \mathrm{s}$. The droplet positions at injection are randomly drawn over the entire slot, resulting in a statistically homogeneous distribution. This operating condition ensures that the liquid phase is not fully evaporated before reaching the flame. The injection velocity of the liquid phase is identical to that of the gas phase, $\boldsymbol{u}^{F}=\boldsymbol{u}_{d}^{F}$. At the fuel side, a turbulence velocity profile is prescribed to assess the effects of turbulence on the evaporation, mixing, and subsequent combustion. The injection of the turbulent velocity field is accomplished by superimposing a synthetic turbulence field over the mean injection velocity, following the Taylor hypothesis Details on the parameters are given in the next section.

The oxidizer side is composed of hot air at $T^{O}=1500 \mathrm{~K}$. The elevated temperature is representative of the recirculation region in gas-turbine combustors to ensure flame stabilization. The injection velocity is prescribed from the constraint of equal gaseous mass-flow rates at both injection streams: $u^{O}=-\frac{T^{O}}{T^{F}} u_{d}^{F}$.

\subsection{Non-dimensional parameters}

The injection velocity on the fuel side is set to $u^{F}=1.0 \mathrm{~m} / \mathrm{s}$, resulting in a global strain rate of $600 \mathrm{~s}^{-1}$, which is comparable to experiments at laminar conditions [16]. The turbulent Reynolds number on the fuel side of the turbulence field that is injected at the fuel side is $\operatorname{Re}_{t}=50$, and this turbulence field is generated by separately performing a simulation of a homogeneous isotropic turbulence satisfying a Passot-Pouquet spectrum [17]. With the present conditions for temperature and global equivalence ratio the turbulent Damköhler and Karlovitz numbers are, respectively, $\mathrm{Da}_{t}=\tau_{L} / \tau_{c}=4.35$ and $\mathrm{Ka}_{t}=\tau_{c} / \tau_{K}=2.43$ where $\tau_{c}$ is the chemical time, $\tau_{K}$ is the Kolmogorov time scale and $\tau_{L}$ is the integral time scale. This combination of parameters indicates potential scale interactions between the small scales of the turbulence and the reaction zones. For the droplet phase, three droplet diameters with $d=20$, 40 and $80 \mu \mathrm{m}$ are investigated, referred to as D20, D40 and D80. These conditions are representative for aeronautical 
combustor applications $[18,19]$, resulting in the Stokes numbers with respect to the Kolomogorov time scale $\tau_{K}$ of $\mathrm{St}_{K}=\tau_{d} / \tau_{k}=1.0,4.0$ and 16.0, respectively. The corresponding Stokes numbers with respect to the global strain rate $a$ are $\mathrm{St}_{a}=a \tau_{d}=0.49,1.98$, and 7.80. Thus, preferential concentration effects are expected and droplets of $d=40$ and $80 \mu \mathrm{m}$ are expected to cross the stagnation plane if they do not evaporate. The initial droplet spacing is $0.25,0.5$ and $1.10 \mathrm{~mm}$ respectively, ensuring sufficient scale separation.

Following the scale analysis, the Kolmogorov length scale is of the order of magnitude of the droplet interspacing. Therefore, we can expect interactions between the evaporation-drag processes and the turbulence [2]. However, these effects are not taken into account in the closure models used here. This question is outside the scope of the present investigation, and will require further analysis. Consequently, the present simulations are referred to as mesoscale/flowfield resolved simulations [20]: given mesoscale closures (evaporation, drag force, combustion), our simulations are fully resolved.

In the following, the three cases are identified by the droplet diameter. In additional, a gas-phase DNS at identical injection conditions and global composition is performed for comparison, and referred to as GAS.

\section{Results}

Computations where performed over $50 \mathrm{~ms}$, with an initialization phase of $20 \mathrm{~ms}$, and a statistical analysis phase of $30 \mathrm{~ms}$. In Figure 2, comparisons of instantaneous temperature fields at the center plane $y=0$ are presented for the gaseous and the liquid injection cases. For the case GAS, the fuel is already injected in gaseous form, so that the mixture fraction $Z$, which is here defined following Bilger's formulation [21], decreases from the fuel side to the oxidizer side. ${ }^{1}$ The flame front is wrinkled by the turbulence and the flame thickness varies depending on the local instantaneous stretch. For the case D20, the mixture fraction increases first due to the liquid fuel evaporation, is first generated by the evaporation of the liquid fuel and then decreases because of mixing between fuel and oxidizer streams. The flame is located after the evaporation zone, and the structure is similar to that of the gaseous flame. For D40, the flame structure is more complex: while the overall structure is similar to that of GAS and D20, isolated reaction zones can be found upstream of the coherent flame front, and will be further analyzed. For the case D80, the flame structure is considerably different from the previous cases. Specifically, the distinct formation of two coherent reaction zones is evident. The first one is located after the evaporation line, and the second one is found on the oxidizer side. Between both zones is a high-temperature region without significant chemical reaction. This region is occupied by large droplets that did not evaporate in the first reaction zone, and will eventually evaporate in the second reaction zone.

\footnotetext{
${ }^{1}$ Since non-equal species diffusivities are considered, Bilger's mixture fraction is not monotonic.
} 
Since the cases D20 and D40 are close to the critical Stokes number of order unity, segregation effects are preferential concentration is expected [22]. To analyze this effect, we investigate the droplet interspace distribution, by means of a Delaunay triangulation on the particle position. The generated tetrahedrons allow to evaluate the interspace volume between droplets, and thus the interspace distance. In Fig. 3, the droplet interspacing is plotted against the axial position, showing a spreading as the droplets are going in to the flame, identified by high temperature. It is also exhibited in Fig. 4, where the distribution close to the injection $(x>0.9 \mathrm{~mm})$ and in the flame $\left(T_{g}>470 \mathrm{~K}\right)$ are presented. These results show the effect of segregation for both cases, the distribution being larger in the flame, indicating that the droplet distribution is approximately log-normal.

\subsection{Spray flame structure bifurcation and hysteresis}

To further analyze the flame structure, results of the laminar spray flame are used in the following to interpret the turbulent flame behavior. The flame structure for the three considered droplet diameters are presented in Fig. 5. The probability in mixture fraction-temperature space is evaluated for the turbulent flames. Results from this analysis at the mid-plane $y=0$ are plotted in Fig. 6, and these data are collected over 30 uncorrelated DNS snapshots. Results for laminar spray flame calculations have also been added. For the purely gaseous case, the turbulence has only a marginal effect, exhibiting only a marginal scattering around the laminar results. Following classical flamelet theory [23], the turbulent GAS solution can be considered as a collection of laminar one-dimensional flames, connecting the oxidizer side at high temperature with the fuel side at low temperature. The flame structure of the gaseous flame is composed of a primary rich premixed reaction region, for which the mixture fraction is approximately constant while the temperature increases, and a secondary recombination zone between the unburnt species and the products of the premixed flame with the oxidizer stream.

For the spray flame cases, the scenario is more complex. At the fuel injection side(point F), the mixture fraction $Z$ is zero. Then, $Z$ increases through evaporation and subsequently decreases by mixing with the air coming from the oxidizer side (point O). Secondly, compared to the gaseous case, the turbulence strongly affects the flame. For all results, mixture fractions higher than the one reached at the corresponding laminar case are found. This is due to the preferential concentration that has been identified previously: by accumulating droplets, the achievable mixture fraction is higher, leading to a broader scatter of the points in the mixture fraction-temperature space.

Finally, differences in the flame structure between the three spray cases are observed from the scatterplot in Fig. 6. One preferential pathway, i.e., one characteristic flame structure, is obtained for cases D20 and D80. In contrast, two distinct flame-structure profiles can be identified for the case D40. Moreover, D20 and D80 present very different flame structures. 
In analogy with the laminar results presented in Fig. 5 for flame structure in physical space, the flame D20 is composed of an evaporation zone, in which the mixture fraction increases, and a single reaction zone comprising both the fuel oxidation and the recombination of the products with the oxidizer (identified by a peak of $\mathrm{OH}$ mass fraction for the laminar fsolution in Fig. 5). In contrast, the case D80 exhibits two local maxima of temperature, which is characteristic of a double-reaction spray flame structure, identified by two peaks of $\mathrm{OH}$ in the laminar solution in Fig. 5. Indeed, a first reaction zone is found on the fuel side, which is connected to the oxidizer side through a second reaction zone. These different flame structures have already been observed for laminar counterflow flames $[4,24,25,26,27]$. The dependence on the droplet diameter observed here is in agreement with the laminar results in [26]. In both cases, the turbulent spray flame can be represented as a collection of laminar flames, even if the space spanned by the turbulent solution for the D80 flame is significantly reduced compared to the D20 case. This is due to the larger inertia of the droplets inducing weaker segregation effects, as discussed in Fig. 3.

In the D40 case, two possible flame-structure pathways are likely to occur. As such, the overall structure presents both aspects of D20 and D80 flames, that is single-reaction and double-reaction structures. These two flame structures are identified in the instantaneous snapshots in Fig. 2 and could be retrieved from the laminar case, see Fig. 5. Indeed, for this operating conditions $\left(d=40 \mu \mathrm{m}, a=600 \mathrm{~s}^{-1}\right)$ multiple spray flame structures have been observed in the laminar case. The existence of multiple solutions for counterflow spray flames has been previously postulated by Continillo and Sirignano [24] on the basis of the high non-linearity of the spray equations and this has been numerically confirmed by Gutheil [28] for laminar spray flames.

In an attempt to understand why this bimodal structure is particular to the case D40, additional laminar simulations are performed by considering a parametric study of droplet diameter and strain rate values. First, the flame structure dependence on the diameter is studied, at the same global strain rate as the turbulent cases. In Fig. 7a., the mean temperature along the axial position normalized by the value of the gaseous flame, is represented. For the case that multiple solutions are found, the normalized mean temperature is higher for the double-reaction flame (where the two reaction zones are separated by a high temperature region) than for the single-reaction flame, allowing to study the transition between these two possible flame structures. Starting from the single-reaction solution obtained for $d=20 \mu \mathrm{m}$, the droplet diameter is successively increased. A single-reaction flame is detected for $D<60 \mu \mathrm{m}$, after which a bifurcation occurs [29]: the flame structure switches to a double-reaction flame for all diameters up to the studied maximum value of $d=80 \mu \mathrm{m}$. Then, to detect an upper branch of the hysteresis curve, we begin with $d=80 \mu \mathrm{m}$ and successively decrease the droplet diameter. Another bifurcation occurs at $d=20 \mu \mathrm{m}$, for which the double-reaction flame collapses again onto a single-reaction zone. Consequently, the laminar spray flame presents a hysteresis [30] with in droplet diameter, i.e. the solution depends on the history and not only on the boundary 
conditions, as shown in Fig. 7a.

The dependence of the flame structure on the strain rate is also investigated by fixing the droplet diameter to $d=40 \mu \mathrm{m}$. Results for the mean temperature normalized by the corresponding gaseous flame value at the same strain rate are shown in Fig. 7b. In this case, two branches are detected: a lower single-reaction branch is discerned for $a \in[500,1100] \mathrm{s}^{-1}$, and an upper branch that contains single-reaction for small strain rate $a \in[300,400] \mathrm{s}^{-1}$ and double-reaction for higher strain rate. The lower branch is generated by starting from the single-reaction solution at $a=600 \mathrm{~s}^{-1}$, and the second branch is obtained by starting either from the lower $\left(a=300 \mathrm{~s}^{-1}\right)$ or the higher $\left(a=1350 \mathrm{~s}^{-1}\right)$ strain rate. Compared to the diameter variations, there is no hysteresis loop for strain-rate variations. Starting from the lower branch, the solution can bifurcate to the upper branch for low $\left(a<500 \mathrm{~s}^{-1}\right)$ or high $(a>$ $1100 \mathrm{~s}^{-1}$ ) strain rates. However when a solution is on the upper upper branch, there is no way to go back to the lower one by varying the strain rate.

A complete diagram of the laminar counterflow spray flame structure is presented in Fig. 8 as a function of the injection droplet diameter and the global strain rate. Single-reaction and double-reaction flames are represented by open and closed symbols, respectively. The bimodal shaded region identifies the hysteresis for which multiple solutions are observed. It explains the bimodal structure of D40 and the unimodal results for D20 and D80, since turbulence induces local variations of the strain rate and droplet diameters at the flame front. Compared to the cases D20 and D80, the D40 flame is more sensitive to such oscillations, showing a bimodal character in analogy to the laminar behavior.

Being able to integrate this bimodal character into turbulent combustion models appears to be challenging, since the spray flame structure is highly sensitive to conditions of the spray and the flow, i.e. strain rate and droplet diameter as well as their history, which could be strongly affected by turbulent combustion models. For instance, flamelet tabulation methods $[31,32,33]$ require recognizing all possible states for each flamelet, which necessitates introducing additional parameters that identify each possible pathway.

\section{Conclusions}

In the present work, a canonical DNS configuration was introduced to study turbulent spray flames at statistically stationary conditions over a wide range of parameters and operating conditions, where the reaction was described using a 24-species reduced mechanism for dodecane.

A parametric study was conducted to systematically examine effects of the turbulence interaction with the liquid spray phase and the flame. Segregation effects due to turbulent mixing were characterized using a Delaunay triangulation to evaluate the droplet interspacing. It was also shown that preferential concentration affects the local 
mixture-fraction field. The flame structure was analyzed, showing that this preferential concentration allows to span a larger mixture-fraction space. We have also observed a regime for which the turbulent flame can be mapped by a collection of laminar flamelets, indicating that turbulent spray flames can be represented this way. Moreover, the sensitivity of the flame structure with respect to droplet diameter as well as local strain rate was identified. From this study, a local bifurcation-mode was identified which arises from the sensitivity to variations in strain rate and diameter variations. The hysteresis of the spray flame structure due to droplet diameter variations has also been observed for laminar cases. Potential issues of the presence of these bifurcations for industrial applications and associated challenges for the modeling of turbulent spray flames were discussed.

\section{Acknowledgements}

The authors thanks H. Pitsch for permission to use the 3DA code, and to greatly acknowledge Shashank and J. Urzay for help on the code. This work used the Extreme Science and Engineering Discovery Environment (XSEDE), which is supported by National Science Foundation grant number ASC130004, and resources of the National Energy Research Scientific Computing Center, which is supported by the Office of Science of the U.S. Department of Energy under Contract No. DE-AC02-05CH11231. The work at the University of Connecticut was supported by the Air Force Office of Scientific Research under Grant FA9550-13-1-0057.

\section{References}

[1] G. M. Faeth, Prog. Energy Comb. Sci. 9 (1983) 1-76.

[2] W. A. Sirignano, Fluid dynamics and transport of droplets and sprays, $2^{\text {nd }}$ edition, Cambridge University Press, 2010.

[3] P. Jenny, D. Roekaerts, N. Beishuizen, Progress in Energy and Combustion Science 38 (2012) 846 - 887.

[4] H. Watanabe, R. Kurose, S. M. Hwang, F. Akamatsu, Combust. Flame 148 (2007) 234-248.

[5] Martinez-Ruiz, D. and Urzay, J. and Sánchez, A. L. and Liñán, A. and Williams, F. A., J. Fluid Mech. (2013) $387-423$.

[6] P. Domingo, L. Vervisch, J. Réveillon, Combust. Flame 140 (2005) 172-195.

[7] J. Réveillon, L. Vervisch, J. Fluid Mech. 537 (2005) 317-347.

[8] Fujita, A. and Watanabe, H. and Kurose, R. and Komori, S., Fuel (2013) 515-525.

[9] Luo, K. and Pitsch, H. and Pai, M.G. and Desjardins, O., Proc. Combust. Inst. (2011) 667-674.

[10] R. Miller, J. Bellan, J. Fluid Mech. 384 (1999) 293-338.

[11] B. Sirjean, E. Dames, D. A. Sheen, H. Wang, in: presented at the 6th US National Combustion Meeting, Ann, Arbor, Michigan. Paper 23 F1.

[12] X. You, F. N. Egolfopoulos, H. Wang, Proc. Combust. Inst. 32 (2009) 403-410.

[13] H. Wang, X. You, A. V. Joshi, S. G. Davis, A. Laskin, F. Egolfopoulos, C.-K. Law, USC Mech Version II., 2007. Available at http: //ignis.usc.edu/USC_Mech_II.htm.

[14] T. Lu, C. K. Law, Prog. Energy Comb. Sci. 35 (2009) 192-215.

[15] Shashank, High fidelity simulation of reactive liquid fuel jets, Ph.D. thesis, Stanford University, 2012. 


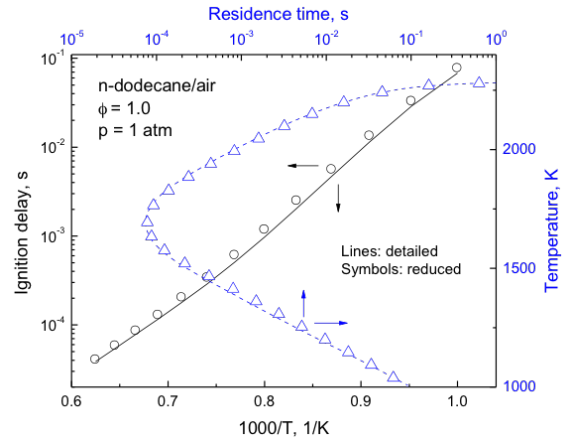

Figure 1: Ignition delay as a function of initial temperature in constant-pressure auto-ignition (bottom-left) and temperature as a function of residence time in PSR with inlet temperature of $300 \mathrm{~K}$ (top-right), for stoichiometric ndodecane/air at atmospheric pressure, calculated with the detailed and reduced mechanisms, respectively.

[16] S. Li, Prog. Energy Comb. Sci. 23 (1997) 303-347.

[17] T. Passot, A. Pouquet, J. Fluid Mech. 181 (1987) 441-466.

[18] R. Lecourt, G. Linassier, G. Lavergne, in: Proceedings of ASME Turbo Expo 2011: Power for Land, Sea and Air, Vancouver, Canada.

[19] T. Providakis, L. Zimmer, P. Scouflaire, S. Ducruix, J. Eng. Gas Turb. and Power 134 (2012).

[20] R. O. Fox, Annual Review of Fluid Mechanics 44 (2012) 47-76.

[21] R. W. Bilger, B. Yip, M. B. Long, A. R. Masri, Combust. Sci. Tech. 72 (1990) 137-155.

[22] K. D. Squires, J. Eaton, J. Fluid Mech. 226 (1991) 1-35.

[23] N. Peters, Prog. Energy Comb. Sci. 10 (1984) 319-339.

[24] G. Continillo, W. Sirignano, Combust. Flame 81 (1990) 325 - 340.

[25] J. B. Greenberg, N. Sarig, Combust. Flame 104 (1996) 431-459.

[26] E. Gutheil, W. A. Sirignano, Combust. Flame 113 (1998) 92-105.

[27] H. Olguin, E. Gutheil, Combust. Flame 161 (2014) 987 - 996.

[28] E. Gutheil, Prog. Comput. Fluid Dyn. 5 (2005) 414-419.

[29] M. Hirsh, S. Smale, R. Devaney, Differential Equations, Dynamical Systems, and an Introduction to Chaos, Elsevier, 2013.

[30] M. Brokate, J. Sprekels, Hysteresis and Phase Transitions, volume 121 of Applied Mathematical Sciences, Springer, 2011.

[31] H. Pitsch, N. Peters, Combust. Flame 114 (1998) 26-40.

[32] O. Gicquel, N. Darabiha, D. Thévenin, Proc. Combust. Inst. 28 (2000) 1901-1908.

[33] C. Hollmann, E. Gutheil, Proc. Combust. Inst. 26 (1996) 1731-1738. 


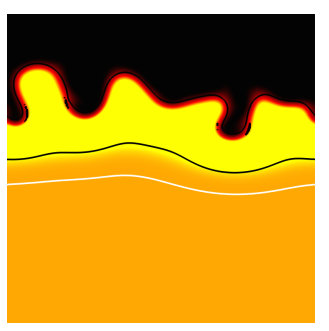

(GAS)

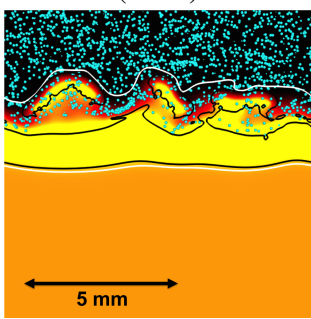

(D40)

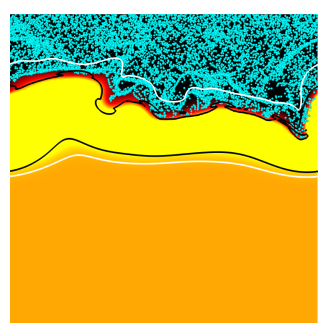

(D20)

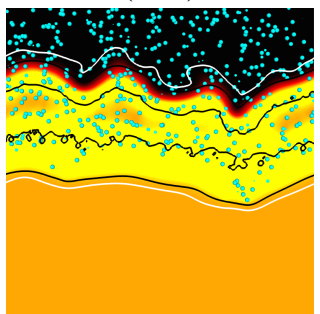

(D80)

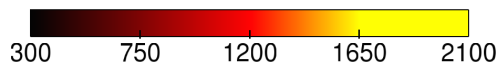

Figure 2: Instantaneous results at the cut plane at $y=0$ : Temperature for GAS, D20, D40 and D80. Isoline of mixture fraction $(Z=0.001$,white $)$ and of heat release $\left(H_{R}=2.10^{7} \mathrm{~J} / \mathrm{kg}\right.$, black). The points represent the droplet positions.

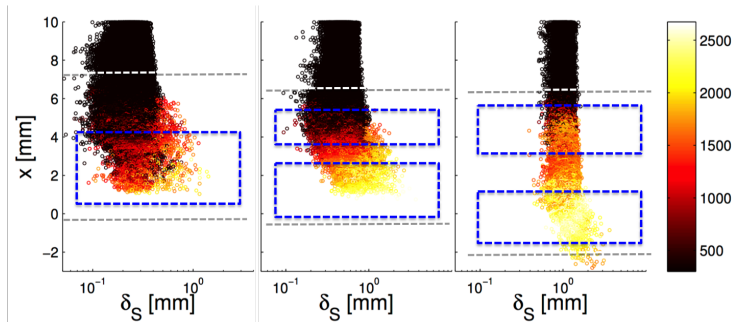

Figure 3: (a) Droplet interspacing based on Delaunay Triangulation versus axial position, colored by gaseous temperature at droplet location, for D20 (left), D40 (center) and D80 (right). The black symbols corresponds to droplet positions for which the gas temperature is less than their boiling temperature, thus delimiting the flame position. The white lines correspond to the mean isoline of mixture fraction $(\mathrm{Z}=0.001)$ and the blue squares identify mean reaction zones $\left(H_{R}>2.10^{7} \mathrm{~J} / \mathrm{kg}\right)$.
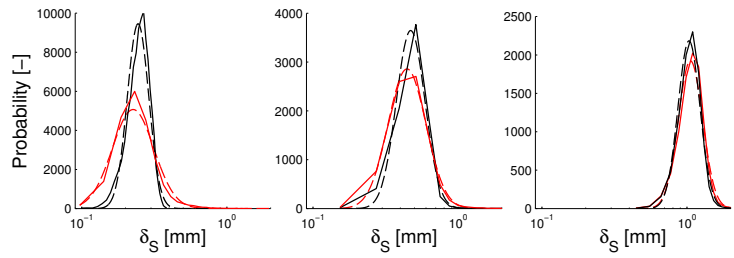

Figure 4: Probability of droplet interspacing close to injection (black line) and in the flame (red lines) for D20 (left), D40 (center) and D80 (right). The dashed line corresponds to the log-normal fitting. 


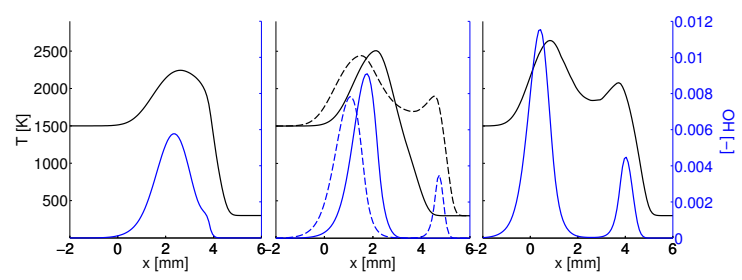

Figure 5: Flame structure for the laminar spray cases: gas temperature (black left axis) and $\mathrm{OH}$ mass fraction (blue right axis) for case D20 (left), D40 (center) and D80 (right). For D40, the single-reaction solution is in full line, the double-reaction in dashed lines.
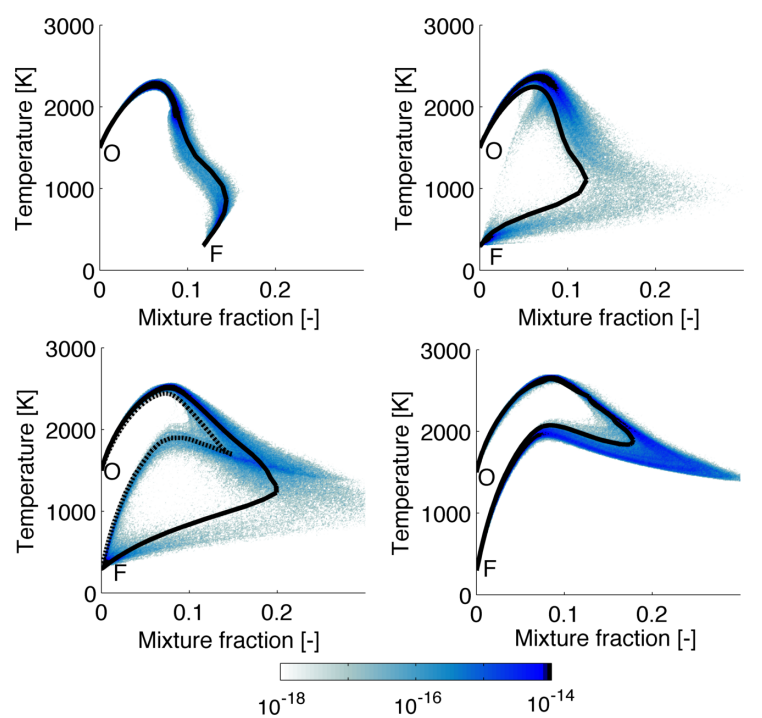

Figure 6: Instantaneous temperature in mixture fraction-temperature space colored by the probability density. Results at the center plane: GAS (upper left), D20 (upper right), D40 (lower left), D80 (lower right). Black lines correspond to the laminar spray-flame solutions of Fig. 5.
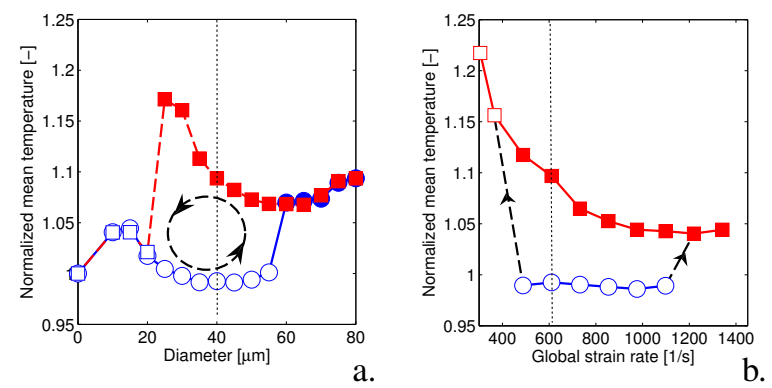

Figure 7: Normalized mean temperature over the axial position at $y=0$ for laminar conditions. In (a), increasing (square with blue line) and decreasing (circles with red line) injection droplet diameter are considered. In (b), strain rate variations are considered, that exhibit a single-reaction branch (blue line) that can bifurcates (black line) into a stable branch (red line) with either single-reaction or double-reaction flame structure. Open and closed symbols denote single-reaction and double-reaction flames, respectively. The vertical lines identify the reference boundary conditions. 


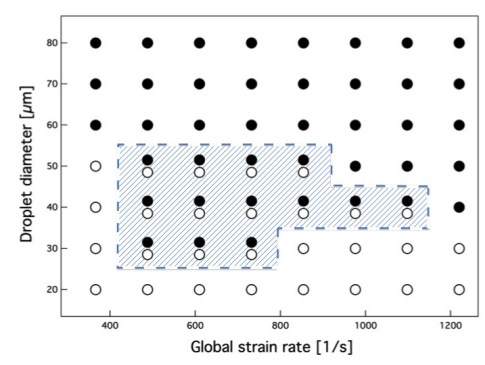

Figure 8: Structure diagram for laminar counterflow spray flames as function of the injection droplet diameter and global strain rate. Open and closed symbols are for single-reaction and double-reaction flames, respectively. The shaded area identifies the bi-modal region. 